\title{
A study of the physical activity restriction in the cancer patients using hierarchical regression analysis
}

\author{
Ji-Youn Kim', Sang-Wan Jeon' ${ }^{1}$ Eun-Surk Yi** \\ 'Exercise Rehabilitation Convergence Institute, Gachon University, Incheon, Korea \\ ${ }^{2}$ Department of Exercise Rehabilitation \& Welfare, College of Health Science, Gachon University, Incheon, Korea
}

This study aimed to examine and analyze the relationship between the physical activity of cancer patients during the hospitalization and the change in exercise recognition and restrictions on physical activity in depth. In this study, adult cancer patients aged more than 20 years residing in the metropolitan area (such as Seoul, Gyeonggi, and Incheon) were selected as a population, and 194 cancer patients from five general hospitals located in metropolitan area were selected as subjects by the purposive sampling. The relative importance for the effect on the physical restriction was greater in the order of the time spent in sedentary activities and exercise positive recognition. The relative importance for cognitive psychological restriction was greater in the order of negative exercise recognition, positive exercise recognition, the time spent in sedentary activities, the time spent in ordinary daily activities and the time spent in intentional movement. In the hospital environment restriction, the facility had a statistically significant effect on the movement and positive exercise recognition. But, socio-cultural restrictions were not affected by positive exercise recognition. As a result of this study, it was found that the physical activity restrictions of the cancer patients may affect differently depending on the physical activity level, exercise experience, and exercise recognition, requiring the interpretation of the physical activity restrictions in various ways.

Keywords: Hospitalized cancer patients, Physical activity restriction, Hierarchical regression analysis

\section{INTRODUCTION}

Statistics Korea reported that the cancer has been the leading cause of death in Korea over the past 34 years. What is worse is that that the number of those who die of cancer is much greater than the number of those who dies of other diseases, which is attributable to $10 \%$ annual increase in the number of cancer patients due to aging, westernized eating habit, and changes in life styles (Statistics Korea, 2018). This high incidence of cancer not only increases national medical expenses but also causes pain to individual patients, aggravating social confusion. Thus, we should put in a great deal of effort to construct an environment to prevent cancers and their recurrence, in addition to treating them effectively.

The healing environment contributes to the stability of body and mind of patients by providing positive factors for promoting the recovery of patients and the factors of the healing environment include overall factors regarding the conditions of hospital such as confidence in medical teams, improvement of hospital service, introduction of new programs, and space utilization. Recently, hospitals and health care facilities located both at home and abroad make efforts to improve facilities for disease prevention and recovery as well as treating diseases, which is the primary purpose of hospitals, without being complacent with medical team-centered hospitals or function-oriented spatial composition (Beauchemin and Hays, 1996). For these changes in healing environment, human motion, that is, physical activity, plays a pivotal role. Johan Huizinga, a cultural anthropologist, mentioned the intrinsic value of motion through a message, "The human motion is an instinct, and has a natural healing power," 'Homo Ludens' (De Groot,
${ }^{*}$ Corresponding author: Eun-Surk Yi (iD https://orcid.org/0000-0002-9370-5231 Department of Exercise Rehabilitation \& Welfare, College of Health Science, Gachon University, 191 Hambangmoe-ro, Yeonsu-gu, Incheon 21936, Korea Tel: +82-32-820-4442, Fax: +82-32-820-4449, E-mail: Yies@gachon.ac.kr Received: August 3, 2018 / Accepted: September 16, 2018
This is an Open Access article distributed under the terms of the Creative Commons Attribution Non-Commercial License (http://creativecommons.org/licenses/by-nc/4.0/) which permits unrestricted non-commercial use, distribution, and reproduction in any medium, provided the original work is properly cited. 
2017). Thus, motion is an essential factor for living to keep and improve our health, and exerts a great influence on the quality of human life (Davies et al., 2010). Moreover, it has been recognized as a crucial factor for patients facing a diversity of diseases, especially in the process of medical service. Basically, physical activity, like motion, has properties to maintain and improve our physical and mental conditions. Specifically, previous studies revealed that the effect of exercise contributes to recovery of cancer patients (Clark et al., 2008; Martin et al., 2009; Meyerhardt et al., 2006; Peddle et al., 2008). As such, physical activity is currently considered more valuable. However, despite of positive effects of physical activity, there are many restrictions on physical activities of hospitalized patients due to spatial limitation of hospitals. The spatial limitation of hospitals imposes restrictions on daily lives of patients, resulting in a significant decline in their physical functions and activities when they are hospitalized (Covinsky et al., 2011). Furthermore, in most cases, physical activity and motion not only do not constitute a large part of medical service for treatment and recovery, but also are excluded because of diverse reasons. Yang et al. (2015) reported that professional medical team recognized the positive effect of rehabilitation treatment with physical motion on the recovery of cancer patients, but in fact they failed to perform it due to the spatial limitation of hospitals.

Most of previous studies conducted on cancer patients have focused on suggesting the methods of exercise (e.g., aerobic exercise, muscle strengthening exercise and combined exercise) and verifying the effects of exercise programs (Persoon et al., 2013), and presenting the significance treatment, recovery, and prevention (Oechsle et al., 2014; Wiskemann et al., 2014), but there are not many studies conducted on the analysis of causes of physical activity restriction in terms of patient-oriented health care service. Moreover, there are few data on the detailed characteristics of hospitalized cancer patients and the cause of the restrictions on the physical activity. Thus, this study aimed to examine and analyze the relationship between the physical activity of cancer patients during the hospitalization and the change in exercise recognition and restrictions on physical activity in depth. Especially the results of analyzing the differences after dividing the patients into those who experienced exercise and those who did not will serve as reference data required for newly establishing the health care service of hospitals and patients-centered service. In addition, this study will provide opportunities to reinforce values of the times for medical service by verifying that instinctive motion of humans play a critical role in improving and maintaining their health after clinical treatment and by providing medical service systems capa- ble of strengthening the instinctive motion of humans.

\section{MATERIALS AND METHODS}

\section{Subjects}

In this study, adult cancer patients aged more than 20 years residing in the metropolitan area (such as Seoul, Gyeonggi, and Incheon) were selected as a population, and 250 cancer patients from five general hospitals located in metropolitan area were selected as subjects by the purposive sampling. A questionnaires survey was conducted among the subjects from Mar to May 2017, and the 194 questionnaires were finally used for analysis excluding 56 questionnaires with missing answers or insincere answers. General characteristics of the subjects are listed in Table 1.

\section{Survey tool}

In this study, a questionnaire survey was used to analyze the mechanism of physical activity experience and restrictions in the hospitalized cancer patients in Korea. For the questionnaire for

Table 1. Demographic characteristics

\begin{tabular}{lc}
\hline Variable & No. of cases (\%) \\
\hline Gender & $72(37.1)$ \\
Male & $122(62.9)$ \\
Female & \\
Age & $8(4.1)$ \\
Below 40s & $32(16.5)$ \\
40s & $69(35.6)$ \\
50s & $48(24.7)$ \\
60s & $37(19.1)$ \\
70s or above & \\
Education & $51(26.3)$ \\
Middle school & $93(47.9)$ \\
High school & $39(20.1)$ \\
College & $11(5.7)$ \\
Undergraduate school or higher & \\
Income (KRW) & $81(41.8)$ \\
2 million or below & $46(23.7)$ \\
2-4 million & $44(22.7)$ \\
4-6 million & $23(11.9)$ \\
6 million or above & $168(86.6)$ \\
Marriage & $13(6.7)$ \\
Married & $13(6.7)$ \\
Unmarried & $194(100)$ \\
Others & \\
Total & \\
\hline & \\
KRW Kogn
\end{tabular}

KRW, Korean won (the currency of South Korea). 
exercise recognition, the questionnaire used in the study and the questionnaire used in the study by Zhu (2001) was modified and supplemented to fit to the contents and subjects in this study; for the questionnaire for physical activity restriction, Chang and $Y_{1}$ (2018) modified and supplemented to fit to the contents and subjects in this study. After conducting preliminary questionnaire survey with 60 cancer patients, the researchers excluded items with factor loading of less than 0.5 and finally constructed the questionnaire with a total of 42 items, including 5 items for background, 6 items for medical history, 10 items for exercise recognition, 8 items for environmental restriction, and 13 for personal restrictions. To help subjects understand contents of the questionnaire, the researchers modified and supplemented the contents using easy-to-understand terms and final questionnaire was reviewed by 5 professors in the department of nursing, physical therapy, and exercise rehabilitation.

\section{Reliability and validity of the questionnaire}

Prior to conducting a questionnaire survey, $\mathrm{PhD}$ degree holders in nursing, physical therapy, exercise rehabilitation, medicine, sports sociology, and exercise physiology reviewed and discussed the validity of the contents and the suitability of items through experts meetings at our request. Also, validity of collected data was finally verified using confirmatory factor analysis. First, principal component analysis was conducted to extract constituent factors; Varimax was used as an orthogonal rotation method to simplify factor loadings; and Kaiser-Meyer-Olkin (KMO) measure was used as a reference value for the selection of variables for factor analysis. Bartlett test of sphericity $\left(\chi^{2}\right)$ was also used to verify the suitability of factor analysis used and to identify common factors. For the inclusion criteria for items, only extracted factors with eigenvalue of 1.0 or more were accepted.

As a result of confirmatory factor analysis, for the exercise recognition, it showed KMO of $0.890, \chi^{2}$ of $1,083.397$, eigenvalue of 5.044 in positive exercise recognition, and eigenvalue of 1.170 in negative exercise recognition. For physical activity restriction, it showed KMO of $0.625, \chi^{2}$ of 973.351 , eigenvalue of 2.376 in physical restriction, eigenvalue of 2.179 in cognitive psychological restriction, eigenvalue of 2.017 in socio-cultural restriction, eigenvalue of 2.206 in facility restriction, and eigenvalue of 1.790 in program restriction. Cronbach coefficient alpha was used for reliability analysis. All factor loadings of 0.5 or more were accepted as a reference to select questionnaire items. As a result, five items in physical activity restriction were excluded. All factors except for these secured validity and reliability coefficient (positive exer- cise recognition $=0.927$, negative exercise recognition $=0.761$, facility restriction $=0.657$, program restriction $=0.709$, cognitive psychological restriction $=0.783$, socio-cultural restriction $=0.731$, physical restriction $=0.675$ ).

\section{Data analysis}

This study was conducted to analyze the mechanism of physical activity experience and restriction in hospitalized domestic cancer patients. To this end, the researchers and associate researchers visited the relevant hospitals to ask for a questionnaire survey and explained the purpose of the survey and how to fill out the questionnaire to subjects in detail. This survey employed a self-administered questionnaire. Among the data collected, questionnaires with unreliable answers, redundant answers and omitted answers were excluded. The data which deemed to be reliable were individually coded into the computer, and processed to serve the study purpose using IBM SPSS Statistics ver. 23.0 (IBM Co., Armonk, NY, USA). In addition, the statistical methods such as frequency analysis, exploratory factor analysis, reliability analysis, correlation analysis, and hierarchical multiple regression analysis, were used in this study, and the significance level was set at $P<0.05$.

Table 2. The effects on physical restriction

\begin{tabular}{llccc}
\hline \multirow{2}{*}{ Model } & \multicolumn{1}{c}{ Variable } & \multicolumn{3}{c}{ Physical restriction } \\
\cline { 3 - 5 } & & $\begin{array}{c}\text { Standardized } \\
\text { coefficient }(\beta)\end{array}$ & $t$ & $P$-value \\
\hline $1 \quad$ Time spent being sedentary & 0.359 & 5.291 & 0.001 \\
& Time spent in daily maintenance & -0.122 & -1.772 & 0.078 \\
& Time spent in intentional movement & 0.032 & 0.474 & 0.636 \\
\hline & Time spent being sedentary & 0.362 & 5.248 & 0.001 \\
& Time spent in daily maintenance & -0.117 & -1.684 & 0.094 \\
Time spent in intentional movement & 0.025 & 0.355 & 0.723 \\
Exercise period & -0.020 & -0.156 & 0.876 \\
Exercise frequency & 0.010 & 0.070 & 0.944 \\
Exercise intensity & 0.056 & 0.395 & 0.693 \\
Time spent being sedentary & 0.343 & 5.168 & 0.001 \\
Time spent in daily maintenance & $0-.085$ & -1.256 & 0.211 \\
Time spent in intentional movement & 0.061 & 0.891 & 0.374 \\
Exercise period & 0.010 & 0.078 & 0.938 \\
Exercise frequency & -0.013 & -0.090 & 0.928 \\
Exercise intensity & 0.163 & 1.176 & 0.241 \\
Positive recognition & -0.273 & -3.846 & 0.001 \\
Negative recognition & 0.111 & 1.664 & 0.098 \\
\hline
\end{tabular}

Model 1: physical activity level, $F=9.876^{* * *}, R^{2}=0.121, D-W=1.512$; model 2: physical activity+exercise experience, $F=4.959^{* * *}, R^{2}=0.110, D-W=1.512$; model 3: physical activity+exercise experience+exercise recognition, $F=6.342^{* * *}, R^{2}=$ $0.181, D-W=1.512$.

${ }^{* * *} P<0.001$. 


\section{RESULTS}

\section{Hierarchical regression analysis of the effect of physical activity, exercise experience, and exercise recognition on physical activity restriction}

Prior to conducting hierarchical regression analysis to investigate the effect of physical activity, exercise experience, exercise recognition on physical activity restriction, the researchers conducted residual analysis of the regression model. The results showed that there were no outliers and that the model did not violate normality, homoscedasticity, and linearity of the residual. In the regression model of physical activity for model 1 (physical activity level), model 2 (physical activity+exercise experience), model 3 (physical activity+exercise experience+exercise recognition), $F$-values were found statistically significant as shown in Tables 2-5. Thus, the model was considered appropriate because it had linearity.

First, the effects on physical restriction are shown in Table 2. The time spent in sedentary activities had a significant positive effect on physical restriction $(\beta=0.359, P<0.001)$. The explanation power for the effect of physical activity on physical restriction

Table 3. The effects on cognitive psychological restriction

\begin{tabular}{llccc}
\hline \multirow{2}{*}{ Model } & \multicolumn{1}{c}{ Variable } & \multicolumn{3}{c}{ Cognitive psychological restriction } \\
\cline { 3 - 5 } & & $\begin{array}{c}\text { Standardized } \\
\text { coefficient }(\beta)\end{array}$ & $t$ & $P$-value \\
\hline $1 \quad$ Time spent being sedentary & 0.297 & 4.407 & 0.001 \\
& Time spent in daily maintenance & 0.095 & 1.391 & 0.166 \\
& Time spent in intentional movement & -0.207 & -3.032 & 0.003 \\
& Time spent being sedentary & 0.284 & 4.305 & 0.001 \\
& Time spent in daily maintenance & 0.072 & 1.074 & 0.284 \\
Time spent in intentional movement & -0.161 & -2.397 & 0.018 \\
Exercise period & 0.003 & 0.020 & 0.984 \\
Exercise frequency & -0.091 & -0.642 & 0.521 \\
Exercise intensity & -0.189 & -1.390 & 0.166 \\
Time spent being sedentary & 0.223 & 5.422 & 0.001 \\
Time spent in daily maintenance & 0.137 & 3.277 & 0.001 \\
Time spent in intentional movement & -0.121 & -2.871 & 0.005 \\
Exercise period & 0.010 & 0.129 & 0.898 \\
Exercise frequency & -0.114 & -1.286 & 0.200 \\
Exercise intensity & 0.063 & 0.730 & 0.467 \\
Positive recognition & -0.367 & -8.359 & 0.001 \\
Negative recognition & 0.606 & 14.655 & 0.001 \\
\hline
\end{tabular}

Model 1: physical activity level, $F=10.557^{* * *}, R^{2}=0.129, D-W=2.118$; model 2: physical activity+exercise experience, $F=8.416^{* * *}, R^{2}=0.187, D-W=2.118$; model 3: physical activity+exercise experience+exercise recognition, $F=53.887^{* * *}, R^{2}=$ $0.687, D-W=2.118$

${ }^{* * *} P<0.001$. was $12.1 \%$. After the addition of the variable of exercise experience, the results of hierarchical regression analysis showed that physical activity and exercise experience had a slightly less effect on physical restriction in model 2 compared to model 1 . For the relative importance of the effect, only the time spent in sedentary activities had a significant effect on physical restriction $(\beta=$ 0.362). Consequently, the explanation power for the effect of physical activity and exercise experience on physical restriction was $11.0 \%$. After the addition of the variable of exercise recognition, the results of hierarchical regression analysis showed that physical activity, exercise experience, and exercise recognition had a greater effect on physical restriction in model 3 compared model 2. The time spent in sedentary activities $(\beta=0.343)$ and positive exercise recognition $(\beta=-0.273)$ affected it differently depending on importance. Consequently, the explanation power for the effect of physical activity, exercise experience, and exercise recognition on physical restriction was $18.1 \%$.

Second, the effects on cognitive psychological restriction are shown in Table 3 . The time spent in sedentary activities $(\beta=$ $0.297)$ and time spent in intentional movement $(\beta=-0.207)$ had significant positive and negative effect on cognitive psychological

Table 4. The effects on socio-cultural restriction

\begin{tabular}{llccc}
\hline \multirow{2}{*}{ Model Variable } & \multicolumn{3}{c}{ Socio-cultural restriction } \\
\cline { 2 - 4 } & & $\begin{array}{c}\text { Standardized } \\
\text { coefficient }(\beta)\end{array}$ & $t$ & P-value \\
\hline $1 \quad$ Time spent being sedentary & 0.100 & 1.407 & 0.161 \\
& Time spent in daily maintenance & 0.133 & 1.846 & 0.066 \\
& Time spent in intentional movement & -0.132 & -1.830 & 0.069 \\
\hline Time spent being sedentary & 0.092 & 1.297 & 0.196 \\
Time spent in daily maintenance & 0.116 & 1.618 & 0.107 \\
Time spent in intentional movement & -0.094 & -1.305 & 0.194 \\
Exercise period & -0.076 & -0.566 & 0.572 \\
Exercise frequency & -0.101 & -0.660 & 0.510 \\
Exercise intensity & -0.046 & -0.312 & 0.755 \\
Time spent being sedentary & 0.083 & 1.183 & 0.238 \\
Time spent in daily maintenance & 0.137 & 1.915 & 0.057 \\
Time spent in intentional movement & -0.068 & -0.946 & 0.345 \\
Exercise period & -0.051 & -0.382 & 0.703 \\
Exercise frequency & -0.119 & -0.783 & 0.434 \\
Exercise intensity & 0.016 & 0.110 & 0.912 \\
Positive recognition & -0.192 & -2.546 & 0.012 \\
Negative recognition & 0.022 & 0.307 & 0.759 \\
\hline
\end{tabular}

Model 1: physical activity level, $F=2.830^{*}, R^{2}=0.043, D-W=2.083$; model 2: physical activity+exercise experience, $F=2.923^{* *}, R^{2}=0.056, D-W=2.083$; model 3 : physical activity+exercise experience+exercise recognition, $F=3.084^{* *}, R^{2}=0.080$, $D-W=2.083$.

${ }^{*} P<0.05 .{ }^{* *} P<0.01$. 
Table 5. The effects on facility restriction and program restriction in hospital

\begin{tabular}{|c|c|c|c|c|c|c|c|}
\hline \multirow[b]{2}{*}{ Model } & \multirow[b]{2}{*}{ Independent variable } & \multicolumn{3}{|c|}{ Facility restriction } & \multicolumn{3}{|c|}{ Program restriction } \\
\hline & & $\begin{array}{l}\text { Standardized } \\
\text { coefficient }(\beta)\end{array}$ & $t$ & $P$-value & $\begin{array}{l}\text { Standardized } \\
\text { coefficient }(\beta)\end{array}$ & $t$ & $P$-value \\
\hline \multirow[t]{3}{*}{1} & Time spent being sedentary & 0.173 & 2.572 & 0.011 & 0.076 & 1.087 & 0.278 \\
\hline & Time spent in daily maintenance & -0.156 & -2.278 & 0.024 & -0.136 & -1.907 & 0.058 \\
\hline & Time spent in intentional movement & 0.342 & 5.023 & 0.000 & 0.250 & 3.522 & 0.001 \\
\hline \multirow[t]{6}{*}{2} & Time spent being sedentary & 0.184 & 2.726 & 0.007 & 0.081 & 1.161 & 0.247 \\
\hline & Time spent in daily maintenance & -0.165 & -2.424 & 0.016 & -0.162 & -2.292 & 0.023 \\
\hline & Time spent in intentional movement & 0.323 & 4.704 & 0.001 & 0.268 & 3.761 & 0.001 \\
\hline & Exercise period & 0.038 & 0.292 & 0.770 & -0.088 & -0.659 & 0.511 \\
\hline & Exercise frequency & 0.310 & 2.132 & 0.034 & 0.211 & 1.394 & 0.165 \\
\hline & Exercise intensity & -0.288 & -2.075 & 0.039 & -0.284 & -1.967 & 0.051 \\
\hline \multirow[t]{8}{*}{3} & Time spent being sedentary & 0.192 & 2.938 & 0.004 & 0.056 & 0.836 & 0.404 \\
\hline & Time spent in daily maintenance & -0.193 & -2.911 & 0.004 & -0.146 & -2.149 & 0.033 \\
\hline & Time spent in intentional movement & 0.283 & 4.239 & 0.001 & 0.266 & 3.886 & 0.001 \\
\hline & Exercise period & -0.005 & -0.039 & 0.969 & -0.107 & -0.842 & 0.401 \\
\hline & Exercise frequency & 0.338 & 2.404 & 0.017 & -0.202 & -1.452 & 0.148 \\
\hline & Exercise intensity & -0.364 & -2.670 & 0.008 & -0.202 & -1.452 & 0.148 \\
\hline & Positive recognition & 0.283 & 4.056 & 0.001 & -0.025 & -0.354 & 0.724 \\
\hline & Negative recognition & 0.036 & 0.551 & 0.582 & 0.313 & 4.661 & 0.001 \\
\hline
\end{tabular}

Facility restriction -- model 1: physical activity level, $F=10.768^{* * *}, R^{2}=0.132, D-W=1.716$; model 2: physical activity+exercise experience, $F=6.558^{* * *}, R^{2}=0.147$, $D-W=1.716$; model 3: physical activity+exercise experience+exercise recognition, $F=7.370^{* * *}, R^{2}=0.209, D-W=1.716$.

Program restriction -- model 1: physical activity level, $F=4.957^{* *}, R^{2}=0.058, D-W=1.484$; model 2: physical activity+exercise experience, $F=3.921^{* *}, R^{2}=0.083, D-W=1.484$; model 3: physical activity+exercise experience+exercise recognition, $F=6.023^{* * *}, R^{2}=0.172, D-W=1.484$.

${ }^{* *} P<0.01 .{ }^{* * *} P<0.001$.

restriction, respectively $(P<0.01, P<0.001)$. The explanation power for the effect of physical activity on cognitive psychological restriction was $12.9 \%$. After the addition of a variable, exercise experience level, hierarchical regression analysis physical activity level and exercise experience level had a greater effect on cognitive psychological restriction in model 2 compared to model 1 . The time spent in sedentary activities $(\beta=0.284)$ and time spent in intentional movement $(\beta=-0.161)$ affected it differently depending on importance. The explanation power for this effect was $18.7 \%$. Similarly, after the addition of the variable of exercise recognition, the results of hierarchical regression analysis showed that physical activity, exercise experience, and exercise recognition had a greater effect on cognitive psychological restriction in model 3 compared to model 2. For the relative importance of the effect, negative exercise recognition $(\beta=0.606$ ) had the most significant effect on cognitive psychological restriction, followed by positive exercise recognition $(\beta=-0.367)$, time spent in sedentary activities $(\beta=0.223)$, the time spent in ordinary daily activities $(\beta=0.137)$, and the time spent in intentional movement $(\beta=$ -0.121). And the explanation power for the effect of physical activity level, exercise experience level, and exercise recognition on cognitive psychological restriction was $68.7 \%$.

Third, the effects on socio-cultural restriction are shown in Table 4 . The physical activity did not affect significantly socio-cultural restriction. The explanation power for the effect of physical activity on socio-cultural restriction was $4.3 \%$. After the addition of the variable of exercise experience, the results of hierarchical regression analysis showed that physical activity and exercise experience had a greater effect on socio-cultural restriction in model 2 compared to model 1 . However, these effects were not statistically significant. The explanation power for this effect was $5.6 \%$. Similarly, after the addition of the variable of exercise recognition, the results of hierarchical regression analysis showed that physical activity, exercise experience, and exercise recognition had a greater effect on socio-cultural restriction in model 3 compared to model 2. For the relative importance of the effect, only positive exercise recognition had a significant effect on socio-cultural restriction $(\beta=0.192)$, and the explanation power for the effect of physical activity, exercise experience, and exercise recognition on socio-cultural restriction was $8.0 \%$.

Fourth, the effects of physical activity constraints on the hospital environment are shown in Table 5. In facility restrictions, 
physical activity and exercise recognition had a greater effect on facility restriction, and the additional exercise recognition had a significant effect on facility restriction (31.3\%) and a positive effect on the time spent in sedentary activities and intentional movement $(P<0.001)$. The relative importance was in the order of the intentional movement $(\beta=0.453)$, and the time spent in sedentary activities $(\beta=0.370)$.

On the contrary, in the facility restriction in the patients who did not experience exercise, the effect of the physical activity and exercise recognition on the facility restriction had a greater effect in model 2 compared to model 1 . And the additional exercise recognition had an effect on the facility restriction (13.9), a positive effect on exercise recognition and the time spent in intentional movement, and a negative effect on the time spent in ordinary daily activities $(P<0.05, P<0.01)$. The relative important was greater in the order of the positive exercise recognition $(\beta=0.311)$, the time spent in daily activities $(\beta=-0.263)$, and the time spent in intentional movement $(\beta=0.218)$.

In program restrictions, the effect of physical activity and exercise recognition had a greater effect on the program restriction in model 2 compared to model 1 , and the additional exercise recognition had an effect on the program restriction (44.6\%), and a positive effect on the time spent in intentional movement and negative exercise recognition and a negative effect on the time spent in ordinary daily activities and positive exercise recognition $(P<0.05, P<0.001)$. The relative importance was greater in the order of the time spent in intentional movement $(\beta=0.496)$, neg- ative exercise recognition $(\beta=0.414)$, the time spent in ordinary daily activities $(\beta=-0.383)$, and positive exercise recognition $(\beta=$ -0.198 ). On the contrary, the effect of physical activity and exercise recognition on the program restriction was greater in model 2 compared to model 1 in the program restriction for the patients who did not experience exercise. And the additional exercise recognition had an effect (6.2\%) on the program restriction, and a positive effect on negative exercise recognition $(P<0.01)$.

\section{Correlation analysis between variables}

Prior to verifying the relationship between variables established in this study, the researchers examined correlation between subvariables and performed correlation analysis to confirm multicollinearity. As a result, there was significant correlation between variables at the level of $P<0.01$ as shown in Table 6. Also, the correlation coefficient between subvariables was found relatively satisfactory as 0.08 or less, indicating there was no problem with multicollinearity between them. These results indicated that each variable was mutually independent.

Specifically, the time spent in sedentary activities had a significant positive correlation with physical restriction, cognitive psychological restriction, and facility restriction. The time spend in ordinary daily activities had a significant positive correlation with the time spent in intentional movement and socio-cultural restriction. The time spent in intentional movement had also a significant positive correlation with exercise period, exercise frequency, exercise intensity, positive exercise recognition, facility restriction,

Table 6. Correlation analysis between variables

\begin{tabular}{|c|c|c|c|c|c|c|c|c|c|c|c|c|c|}
\hline & a & $b$ & c & $d$ & e & $f$ & g & $\mathrm{h}$ & i & j & k & 1 & $\mathrm{~m}$ \\
\hline a & 1 & & & & & & & & & & & & \\
\hline b & 0.088 & 1 & & & & & & & & & & & \\
\hline c & -0.033 & $0.161^{*}$ & 1 & & & & & & & & & & \\
\hline$d$ & -0.004 & -0.072 & $0.135^{*}$ & 1 & & & & & & & & & \\
\hline e & -0.082 & -0.026 & $0.181^{* *}$ & $0.828^{* *}$ & 1 & & & & & & & & \\
\hline$f$ & -0.053 & -0.080 & $0.140^{*}$ & $0.815^{* *}$ & $0.857^{* *}$ & 1 & & & & & & & \\
\hline g & -0.041 & 0.090 & $0.199 * *$ & $0.316^{* *}$ & $0.302^{* *}$ & $0.343^{* *}$ & 1 & & & & & & \\
\hline h & 0.087 & -0.022 & -0.019 & $-0.130^{*}$ & $-0.163^{*}$ & $-0.191^{* *}$ & $-0.120^{*}$ & 1 & & & & & \\
\hline i & $0.286^{* *}$ & -0.119 & -0.128 & -0.082 & -0.126 & -0.073 & $-0.411^{* *}$ & 0.038 & 1 & & & & \\
\hline j & $0.239 * *$ & 0.090 & -0.094 & $-0.319^{* *}$ & $-0.356^{* *}$ & $-0.383^{* *}$ & $-0.380^{* *}$ & $0.740^{* *}$ & $0.180^{* *}$ & 1 & & & \\
\hline k & 0.094 & $0.166^{*}$ & -0.082 & $-0.309^{* *}$ & $-0.284^{* *}$ & $-0.296^{* *}$ & $-0.233^{* *}$ & 0.034 & $0.190^{* *}$ & 0.134 & 1 & & \\
\hline I & $0.142^{*}$ & -0.099 & $0.287^{* *}$ & $0.141^{*}$ & $0.160^{*}$ & 0.076 & $0.245^{* *}$ & 0.049 & 0.068 & -0.126 & -0.031 & 1 & \\
\hline $\mathrm{m}$ & 0.045 & -0.079 & $0.180^{*}$ & -0.020 & 0.057 & -0.019 & -0.031 & $0.251^{* *}$ & -0.047 & 0.009 & 0.030 & $0.230^{* *}$ & 1 \\
\hline
\end{tabular}

a, time spent being sedentary; $b$, time spent in daily maintenance; $c$, time spent in intentional movement; $d$, exercise period; e, exercise frequency; $f$, exercise intensity; $g$, positive exercise recognition; h, negative exercise recognition; i, physical restriction; j, cognitive psychological restriction; k, socio-cultural restriction; I, facility restriction; m, program restriction. ${ }^{*} P<0.05$. ${ }^{*} P<0.01$. 
and program restriction. For exercise period, it had a significant positive correlation with exercise frequency, exercise intensity, positive exercise recognition, facility restriction, but it had a significant negative correlation with negative exercise recognition, cognitive psychological restriction, and socio-cultural restriction. For exercise frequency, it had a significant positive correlation with exercise intensity, positive exercise recognition, and facility restriction, but it had a significant negative correlation with negative exercise recognition, physical restriction, cognitive psychological restriction, and socio-cultural restriction. For the exercise intensity, it had a significant positive correlation with positive exercise recognition, but it had a significant negative correlation with negative exercise recognition, physical restriction, cognitive psychological restriction, and socio-cultural restriction. There was a significant positive correlation between positive exercise recognition and facility restriction, but there was a significant negative correlation between positive exercise recognition and other variables, including negative exercise recognition, physical restriction, cognitive psychological restriction, and socio-cultural restriction. For positive exercise recognition, it had a significant positive correlation with facility restriction, but it had a significant negative correlation with negative exercise recognition, physical restriction, cognitive psychological restriction, and socio-cultural restriction. Negative exercise recognition had a significant positive correlation with cognitive psychological restriction and program restriction. The physical restriction had a significant correlation with cognitive psychological restriction and the facility restriction had a significant correlation with the program restriction.

\section{DISCUSSION}

This study was conducted to empirically investigate the mechanism of physical activity experience and restrictions in hospitalized cancer patients in Korea. To this end, the researchers analyzed how physical activity experience and exercise recognition affect the physical activity restriction of hospitalized cancer patients.

As recent case studies reported that physical activity may be effective in reducing cancer recurrence and in preventing and recovering from cancer, it positively affects the positive exercise recognition by patients (Meyerhardt et al., 2006). On the contrary, the cancer patients tend to not want to move at all due to pain and psychological weakness in the event of cancer recurrence or during treatment. This weak-willed behavior may cause restrictions to exercise recognition (Fadul et al., 2008).

In this study, it was found that in the personal physical activity restriction for the amount of physical activity, exercise experience and exercise recognition, the time spent in sedentary activities had a significant positive effect on physical restriction $(\beta=0.359, P<$ $0.001)$ and cognitive psychological restriction $(\beta=0.297, P<$ 0.001). According to the additional variable of exercise experience, the effect of physical activity and exercise experience on the physical restrictions was slightly decreased in model 2. According to the additional variable of exercise recognition, the effect of physical activity and exercise experience and exercise recognition were increased in model 1 . The relative importance for the effect on the physical restriction was greater in the order of the time spent in sedentary activities $(\beta=0.343)$ and exercise positive recognition $(\beta=-0.273)$. The relative importance for cognitive psychological restriction was greater in the order of negative exercise recognition $(\beta=0.606)$, positive exercise recognition $(\beta=-0.367)$, the time spent in sedentary activities $(\beta=0.223)$, the time spent in ordinary daily activities and the time spent in intentional movement $(\beta=-0.121)$. Unfamiliar behavioral habits and desire to participate in exercise may induce physical restrictions, and in the aspect of socio-cultural restrictions, the changed environment in the hospital may also induce the physical restrictions due to the absence of companion, limitations in the support from other people and the limitations in managers and leaders. In conclusion, such phenomena as social and cultural constraints may occur for these reasons. In addition, the deterioration of physical and mental conditions experienced in during chemotherapy and other medical treatments are considered to limit the establishment of surrounding environment enabling active physical activities before the occurrence of cancer. In prior studies, it was reported the that the physical and mental suffering experienced in the cancer treatment process made it difficult to maintain a certain level of physical condition and to engage in physical activities (Schmitz et al., 2010), supporting the results of this study.

However, socio-cultural restrictions were not affected by positive exercise recognition. In general, forcing cancer patients to exercise themselves can be another cause of stress to them. Therefore, it is important to create an environment in which they can exercise themselves (Mutrie et al., 2007) and to change the exercise recognition in positive way through continued motivation by family, medical team and exercise experts because it is considered to be effective in reducing social-cultural restrictions.

In the hospital environment restriction, the facility had a statistically significant effect on the movement $(\beta=0.342, P<0.001)$ and positive exercise recognition $(\beta=0.283, P<0.001)$, implying that cancer patients suffering from cancer are positively receptive 
to physical activity that may be effective for recovery, and even if the given environment is insufficient, they feel less restrictions by accepting the benefits of exercise through physical activities. In addition, the negative exercise recognition arises because cancer patients feel unfamiliarity in the new environment of the hospital, not the existing exercise environment, even though they have exercise experiences in the past. For example, a negative exercise recognition may be formed by feeling resistance to new exercise methods recommended by doctors or by being conscious of others. Yang et al. (2015) said that that it is a reality that the patients cannot practice physical activities due to special restriction of hospital. In addition, it has been reported that the provision of exercise information from hospitals has been limited to "moderate", limiting the actual practice (Lin et al., 2014). It was found that the effect of positive exercise recognition and negative exercise recognition are inversely related to program restrictions. These results imply that the patients with positive exercise experience tend to rationalize the exercise program provided by the hospital even though it is insufficient, whereas negative exercise recognition adversely affects the exercise program for cancer patients if it does not meet the expectation of the patients. In this regard, Schwartz (1999) and Kim and Yi (2017) reported that the patients were reluctant to participate in the program if their exercise program did not meet the expectations of the patients. Because the patients with serious disease such as cancer have stronger will to extend life expectancy and to recover than patients with general diseases, they tend to have greater expectations on various interventions such as nutrition and supplements including exercise. Therefore there will be restriction on their participation in exercise if their expectations are not satisfied. In particular, the lack of exercise information may limit the participation of patients receiving chemotherapy in the exercise program (Adamsen et al., 2004). On the contrary, the increased recognition on the necessity of health care due to diagnosis with cancer leads to physical activities. The amount of their physical activity is higher than that of normal middle-aged adults (Liou et al., 2011) and the evidence for the effect of physical activity on the prevention of cancer is rapidly increasing, suggesting that the physical activity of cancer patients is attracting attention as a non-pharmacological intervention during hospitalization (Luctkar-Flude et al., 2009).

Finally, as a result of analyzing the correlation with the amount of physical activity during hospitalization, the time spent in sedentary activities affected physical restrictions, cognitive-psychological restrictions and positive exercise recognition, and the time spent in ordinary daily activities and the intentional movement were affected by 'socio-cultural restrictions' and 'positive exercise recognition and the hospital facility' respectively. Hospital facilities were not significantly affected by other variables besides exercise recognition. This implies that the exercise experience, which is used as an important scale to analyze the restriction on leisure participation by the public, can be accepted as a different meaning for the cancer patients suffering from pain. In particular, there is a limit to understanding is importance from the patients in the limited space of hospital.

Segal et al. (2001) reported that cancer patients confirmed advantages of exercise and became more physically active through physician-based assessment and counseling for exercise program, since it puts stress on the cognitive and empirical process in an early stage of exercise, and through this process, the physical activities affected social support and self-efficacy such as resolving problems relating to social support, setting of goals and disability, etc.

As a result of this study, it was found that the physical activity restrictions of the cancer patients may affect differently depending on the physical activity level, exercise experience and exercise recognition, requiring the interpretation of the physical activity restrictions in various ways. Therefore, analyzing the characteristics of hospitalized cancer patients and analyzing why their physical activities necessary for recovery are restricted will provide an opportunity to improve medical services for cancer patients in a practical way, and suggest directions for health and medical care services for environment as well as individuals and also to enhance the values of medical services. It will also provide an opportunity to gain quantitative data to help expand the concept of paradigm shift in medical care service. Based on the results of this study, the researchers suggest that future studies are conducted to analyze the personal characteristics and the hospital environment in detail by developing the evaluation paper that can classify the medical conditions of the hospitalized cancer patient in future and it will be necessary to continue to seek ways to improve physical activity restrictions through convergence with other academic disciplines.

\section{CONFLICT OF INTEREST}

No potential conflict of interest relevant to this article was reported.

\section{ACKNOWLEDGMENTS}

This work was supported by the Ministry of Education of the 
Republic of Korea and the National Research Foundation of Korea (NRF-2016S1A5B6913737).

\section{REFERENCES}

Adamsen L, Midtgaard J, Andersen C, Quist M, Moeller T, Roerth M. Transforming the nature of fatigue through exercise: qualitative findings from a multidimensional exercise programme in cancer patients undergoing chemotherapy. Eur J Cancer Care (Engl) 2004;13:362-370.

Beauchemin KM, Hays P. Sunny hospital rooms expedite recovery from severe and refractory depressions. J Affect Disord 1996;40:49-51.

Chang IY, Yi ES. The influence of environmental constraints within hospitals on physical activity level of cancer patients. J Exerc Rehabil 2018; 14:382-386.

Clark MM, Novotny PJ, Patten CA, Rausch SM, Garces YI, Jatoi A, Sloan JA, Yang P. Motivational readiness for physical activity and quality of life in long-term lung cancer survivors. Lung Cancer 2008;61:117-122.

Covinsky KE, Pierluissi E, Johnston CB. Hospitalization-associated disability: "She was probably able to ambulate, but I'm not sure". JAMA 2011;306:1782-1793.

Davies EJ, Moxham T, Rees K, Singh S, Coats AJ, Ebrahim S, Lough F, Taylor RS. Exercise training for systolic heart failure: Cochrane systematic review and meta-analysis. Eur J Heart Fail 2010;12:706-715.

De Groot K. Bingo! Holy play in experience-oriented society. Soc Compass 2017;64:194-205.

Fadul NA, El Osta B, Dalal S, Poulter VA, Bruera E. Comparison of symptom burden among patients referred to palliative care with hematologic malignancies versus those with solid tumors. J Palliat Med 2008; 11:422-427.

Kim JY, Yi ES. Nationwide survey of cancer center programs in Korea. J Exerc Rehabil 2017;13:300-306.

Lin KY, Shun SC, Lai YH, Liang JT, Tsauo JY. Comparison of the effects of a supervised exercise program and usual care in patients with colorectal cancer undergoing chemotherapy. Cancer Nurs 2014;37:E21-29.

Liou YM, Lee HL, Chien LY, Kao WY, Chiang CC, Wang DY. Daily-life physical activity and related factors among patients with cancer receiving chemotherapy in Taiwan. Cancer Nurs 2011;34:443-452.

Luctkar-Flude M, Groll D, Woodend K, Tranmer J. Fatigue and physical activity in older patients with cancer: a six-month follow-up study. Oncol Nurs Forum 2009;36:194-202.

Martin CK, Church TS, Thompson AM, Earnest CP, Blair SN. Exercise dose and quality of life: a randomized controlled trial. Arch Intern Med 2009;169:269-278.
Meyerhardt JA, Heseltine D, Niedzwiecki D, Hollis D, Saltz LB, Mayer RJ, Thomas J, Nelson H, Whittom R, Hantel A, Schilsky RL, Fuchs CS. Impact of physical activity on cancer recurrence and survival in patients with stage III colon cancer: findings from CALGB 89803. J Clin Oncol 2006;24:3535-3541.

Mutrie N, Campbell AM, Whyte F, McConnachie A, Emslie C, Lee L, Kearney N, Walker A, Ritchie D. Benefits of supervised group exercise programme for women being treated for early stage breast cancer: pragmatic randomised controlled trial. BMJ 2007;334:517.

Oechsle K, Aslan Z, Suesse Y, Jensen W, Bokemeyer C, de Wit M. Multimodal exercise training during myeloablative chemotherapy: a prospective randomized pilot trial. Support Care Cancer 2014;22:63-69.

Peddle CJ, Au HJ, Courneya KS. Associations between exercise, quality of life, and fatigue in colorectal cancer survivors. Dis Colon Rectum 2008; 51:1242-1248.

Persoon S, Kersten MJ, van der Weiden K, Buffart LM, Nollet F, Brug J, Chinapaw MJ. Effects of exercise in patients treated with stem cell transplantation for a hematologic malignancy: a systematic review and meta-analysis. Cancer Treat Rev 2013;39:682-690.

Schmitz KH, Courneya KS, Matthews C, Demark-Wahnefried W, Galvão DA, Pinto BM, Irwin ML, Wolin KY, Segal RJ, Lucia A, Schneider CM, von Gruenigen VE, Schwartz AL; American College of Sports Medicine. American College of Sports Medicine roundtable on exercise guidelines for cancer survivors. Med Sci Sports Exerc 2010;42:14091426.

Schwartz AL. Fatigue mediates the effects of exercise on quality of life. Qual Life Res 1999;8:529-538.

Segal R, Evans W, Johnson D, Smith J, Colletta S, Gayton J, Woodard S, Wells G, Reid R. Structured exercise improves physical functioning in women with stages I and II breast cancer: results of a randomized controlled trial. J Clin Oncol 2001;19:657-665.

Statistics Korea. Disability stats [Internet]. Deajeon (Korea): Statistics Korea; c2018 [cited 2018 Jul 10]. Available from: http://www.index.go.kr/ main.do?cate $=6$.

Wiskemann J, Kuehl R, Dreger P, Schwerdtfeger R, Huber G, Ulrich CM, Jaeger D, Bohus M. Efficacy of exercise training in SCT patients--who benefits most? Bone Marrow Transplant 2014;49:443-448.

Yang EJ, Chung SH, Jeon JY, Seo KS, Shin HI, Hwang JH, Lim JY. Current practice and barriers in cancer rehabilitation: perspectives of Korean physiatrists. Cancer Res Treat 2015;47:370-378.

Zhu W, Timm G, Ainsworth B. Rasch calibration and optimal categorization of an instrument measuring women's exercise perseverance and barriers. Res Q Exerc Sport 2001;72:104-116. 